\title{
Room Temperature Enhancement of Electronic Materials by Superacid Analogues
}

\author{
Sophie L. Pain,* Nicholas E. Grant, and John D. Murphy*
}

Cite This: ACS Nano 2022, 16, 1260-1270

Read Online

\section{ACCESS}

山 Metrics \& More

回国 Article Recommendations

S1 Supporting Information

ABSTRACT: Treatment with the superacid bis(trifluoromethanesulfonyl)amide (sometimes known as TFSA, TFSI, or $\mathrm{HNTf}_{2}$ ) enhances the properties of a wide range of optoelectronic materials, resulting in longer effective carrier lifetimes and higher photoluminescence quantum yields. We have conducted a multimaterial study treating both crystalline silicon and transition metal dichalcogenide (TMDC) monolayers and few-layer flakes with solutions formed from TFSA and a range of compounds with related chemical structures with different Lewis acidities, in order to elucidate the factors underpinning the TFSA-related class of enhancement treatments. We adopt dichloromethane (DCM) as a common solvent
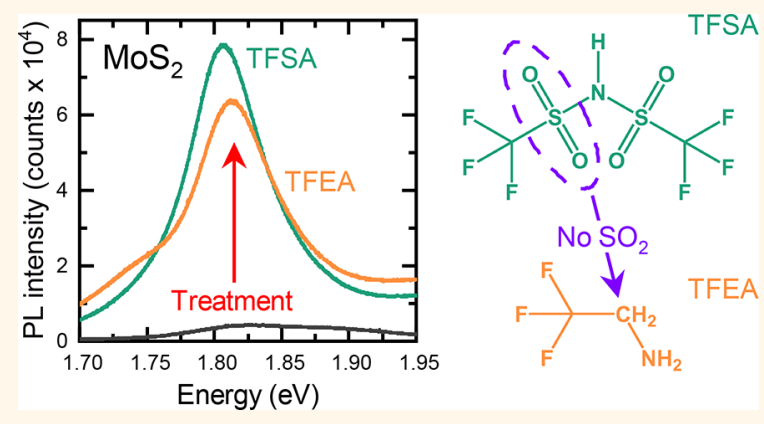
as it provides good results at room temperature and is potentially less hazardous than TFSA-dichloroethane (DCE) heated to $\sim 100{ }^{\circ} \mathrm{C}$, which has been used previously. Kelvin probe experiments on silicon demonstrate that structurally similar chemicals give passivating films with substantially different charge levels, with the higher levels of charge associated with the presence of $\mathrm{CF}_{3} \mathrm{SO}_{2}$ groups resulting in longer effective lifetimes due to an enhancement in field-effect passivation. Treatment with all analogue solutions used results in enhanced photoluminescence in $\mathrm{MoS}_{2}$ and $\mathrm{WS}_{2}$ compared to untreated controls. Importantly we find that $\mathrm{MoS}_{2}$ and $\mathrm{WS}_{2}$ can be enhanced by analogues to TFSA that lack sulfonyl groups, meaning an alternative mechanism to that proposed in computational reports for TFSA enhancement must apply.

KEYWORDS: lifetime, photoluminescence, silicon, superacid, passivation, transition metal dichalcogenides

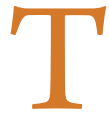

he performance of electronic devices is usually limited by the properties of the material from which they are made. For some devices, such as silicon solar cells, charge carrier lifetime (or diffusion length) is a key parameter as it governs how many carriers are collected. As silicon is relatively defect free, under practical operating conditions, cell efficiency is strongly influenced by recombination at surfaces unless they are adequately passivated. Other devices are intended to emit light. Interestingly, light emission from transition metal dichalcogenides (TMDCs) occurs in materials that have relatively high defect densities yet the photoluminescence (PL) quantum yield in these excitonic materials can be high when the excess charge is suppressed. ${ }^{1,2}$

Wet chemical passivation treatments with the superacid bis(trifluoromethanesulfonyl)amide $\left(\left(\mathrm{CF}_{3} \mathrm{SO}_{2}\right)_{2} \mathrm{NH}, \mathrm{TFSA}\right.$, sometimes TFSI, or $\mathrm{HNTf}_{2}$ ) have recently attracted considerable interest. TFSA-based solutions enhance the PL of TMDCs such as $\mathrm{MoS}_{2}$ and $\mathrm{WS}_{2},{ }^{3-13}$ which have applications in new generations of optoelectronic devices. TFSA-based treatments have also been shown to be extremely effective at passivating crystalline silicon, ${ }^{14-17}$ offering surface recombination velocities $<1 \mathrm{~cm} / \mathrm{s}^{15,16,18}$ (similar to liquid
HF) ${ }^{19}$ and thus being competitive with the best dielectric films ${ }^{20}$ without causing lifetime degradation, which can occur at dielectric deposition temperatures. ${ }^{21}$ There are also indications that TFSA-based treatments of silicon anodes can improve the stability of lithium-ion batteries. ${ }^{22}$

Although the empirical effects of TFSA-based treatments are clear, mechanisms by which they occur are not wellunderstood. What is known is that, for both silicon and TMDCs, the level of enhancement is highly dependent on solvent choice ${ }^{7,16,23}$ surface preparation, and processing, 3 and the beneficial effects degrade under a vacuum. ${ }^{6,17}$ Solution acidity appears to play a role, as Brønsted superacidity is thought to be crucial for the enhancement of TMDCs, $3,7,24$ particularly $\mathrm{MoS}_{2}$, while moderate or high levels of acidity are required for silicon passivation. ${ }^{17,18}$ Additionally, it has been

Received: October 14, 2021

Accepted: December 8, 2021

Published: January 3, 2022 

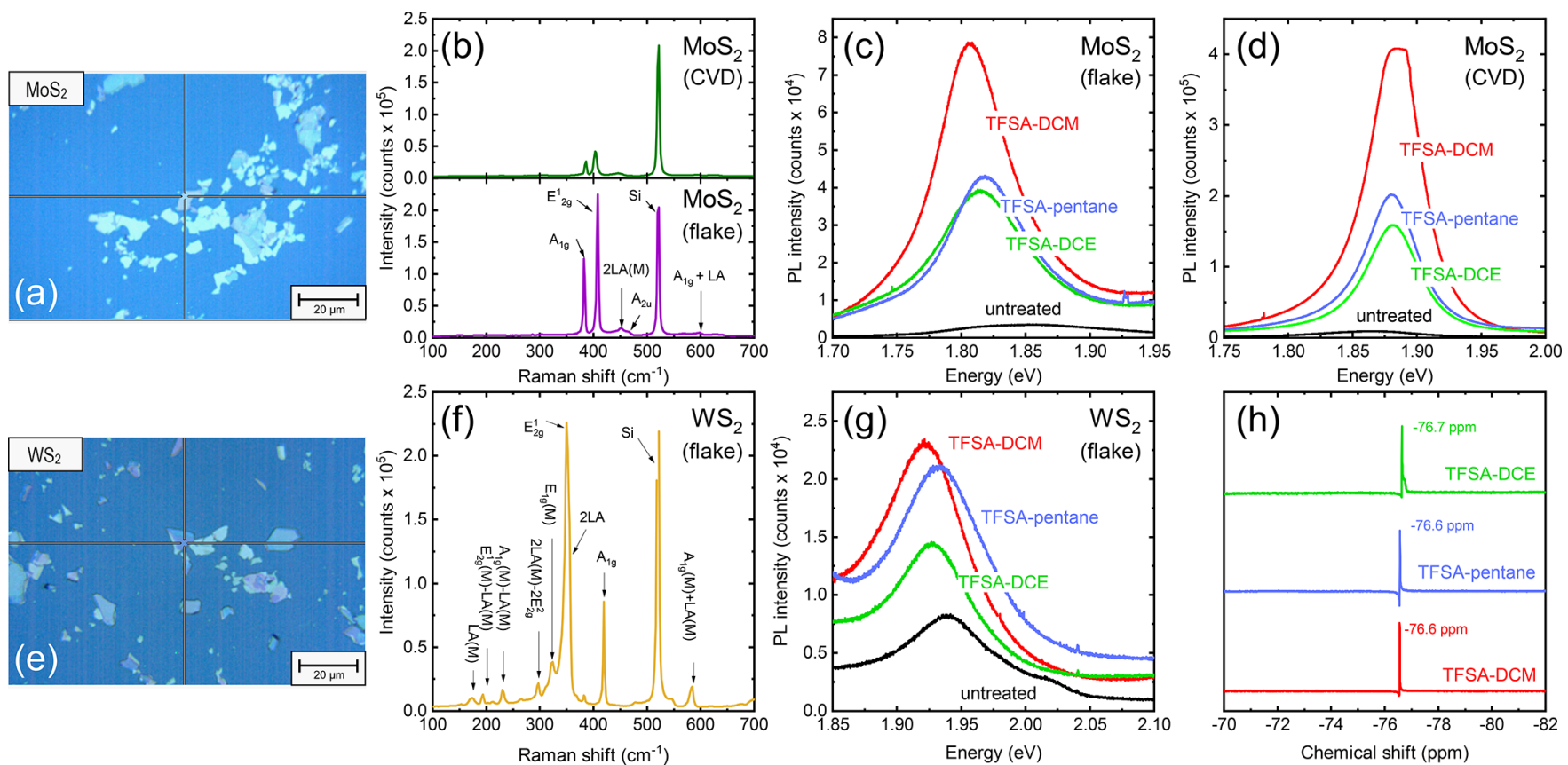

Figure 1. (a) Representative optical micrograph of $\mathrm{MoS}_{2}$ flakes on a $\mathrm{SiO}_{2} / \mathrm{Si}$ surface. (b) Raman spectra of monolayer $\mathrm{CVD} \mathrm{MoS}_{2}$ (top) and few-layer $\mathrm{MoS}_{2}$ flakes (bottom), collected under the same conditions with peak assignments. ${ }^{28}$ (c) PL spectra for few-layer MoS flakes treated with TFSA-DCM, TFSA-DCE, or TFSA-pentane at room temperature, relative to an untreated control. (d) PL spectra for CVDgrown monolayer $\mathrm{MoS}_{2}$ treated with TFSA-DCM, TFSA-DCE, or TFSA-pentane at room temperature, relative to an untreated control. (e) Representative optical micrograph of $\mathrm{WS}_{2}$ flakes on a $\mathrm{SiO}_{2} / \mathrm{Si}$ surface. (f) Example Raman spectrum of $\mathrm{WS}_{2}$ with peak assignments. ${ }^{28,29}$ (g) PL spectra for $\mathrm{WS}_{2}$ flakes treated with TFSA-DCM, TFSA-DCE, and TFSA-pentane at room temperature, relative to an untreated control. (h) ${ }^{19} \mathrm{~F}\left\{{ }^{1} \mathrm{H}\right\}$ NMR spectra of solutions of TFSA in different solvents.

shown that TFSA-based passivation of silicon involves both chemical (termination of dangling bonds) and field (surface charge repelling charge carriers) effects, ${ }^{17}$ while both effects have separately been proposed for TMDCs. ${ }^{12,23,24}$ There have been a number of recent computational studies that conclude that passivation of the sulfur vacancy site is responsible for PL enhancement in $\mathrm{MoS}_{2}$.

In this paper, we aim to elucidate the factors underpinning the TFSA-related class of treatments by performing a direct comparative study between TMDCs and silicon. As well as studying TFSA itself, we develop treatments using a set of analogue solutions based on molecules that have key structural similarities with, and differences to, TFSA. Considering the exceptional behavior of TFSA, other studies have rationalized its chemistry through comparison with structurally related triflic acid, which has previously been shown to passivate silicon, ${ }^{14,26}$ and an analogue approach has recently been taken for understanding the passivation of defects in perovskite photovoltaics. $^{27}$ We perform experiments on both TMDC materials and crystalline silicon, with the latter providing an ideal model system for understanding passivation mechanisms with larger area characterization techniques.

Another aim of this paper is to address an important practical issue affecting enhancement treatments of TMDCs. Superacidic treatments of TMDCs usually require processing at elevated temperatures, ${ }^{3-5,7,9-12}$ with the largest $\mathrm{MoS}_{2}$ enhancement to our knowledge achieved using TFSA in a 1,2-dichloroethane (DCE)/1,2-dichlorobenzene (DCB) solution heated to $100{ }^{\circ} \mathrm{C}$. ${ }^{5}$ As DCE is highly flammable, toxic, mutagenic, potentially carcinogenic, and is discouraged for use at industrial scale, it is necessary to determine if less hazardous solvents and processes could be developed. ${ }^{30,31}$

\section{RESULTS AND DISCUSSION}

We start by presenting data on room temperature TFSA-based enhancement of TMDCs (Figure 1). $\mathrm{MoS}_{2}$ and $\mathrm{WS}_{2}$ flakes were mechanically exfoliated onto $\mathrm{SiO}_{2} / \mathrm{Si}$ substrates, with representative optical micrographs shown in Figure 1a,e. Layer thicknesses were determined from peak separation of $E_{2 g}^{1}$ and $A_{1 g}$ Raman modes, with selected spectra shown in Figure $1 b, f$. From analysis of the Raman spectra, the $\mathrm{MoS}_{2}$ flakes studied were three to four layers thick (average peak separation $\sim 24$ $\mathrm{cm}^{-1}$ ). The $\mathrm{WS}_{2}$ flakes chosen for PL studies were monolayers (average peak separation $\sim 60 \mathrm{~cm}^{-1}$ ). ${ }^{29,32-35}$ A commercially available CVD monolayer $\mathrm{MoS}_{2}$ sample (peak separation $\sim 18$ $\mathrm{cm}^{-1}$ ) was also studied to avoid any ambiguity arising from the differing layer thicknesses of the $\mathrm{WS}_{2}$ and $\mathrm{MoS}_{2}$ flakes.

We investigated the solvent dependence of TFSA enhancement of $\mathrm{MoS}_{2}$ and $\mathrm{WS}_{2}$ with PL results presented in Figure $1 c, d, g$, respectively. Mechanically exfoliated samples were cleaned in acetone-isopropanol prior to measurement, although the PL was insensitive to the type of cleaning used (Figure S1). DCE is a commonly used solvent for TMDC treatments in the literature, $, 3,11,36,37$ and dichloromethane (DCM) and pentane have been shown to be highly effective in solutions used to passivate silicon. ${ }^{16,18}$

For both monolayer and few-layer $\mathrm{MoS}_{2}$, TFSA-DCM and TFSA-pentane treatments outperform TFSA-DCE at room temperature, with the level of $\mathrm{MoS}_{2}$ enhancement achieved by TFSA-DCM roughly twice that achieved by TFSA-DCE. The $\mathrm{PL}$ enhancement trends with solvent are the same for $\mathrm{MoS}_{2}$ in both monolayer and few-layer flake forms, which suggests insights gained from studying flakes are likely to also be applicable to monolayers. The lower relative enhancement in $\mathrm{WS}_{2}$ compared to $\mathrm{MoS}_{2}$ is in keeping with other reports. ${ }^{5}$ Our 

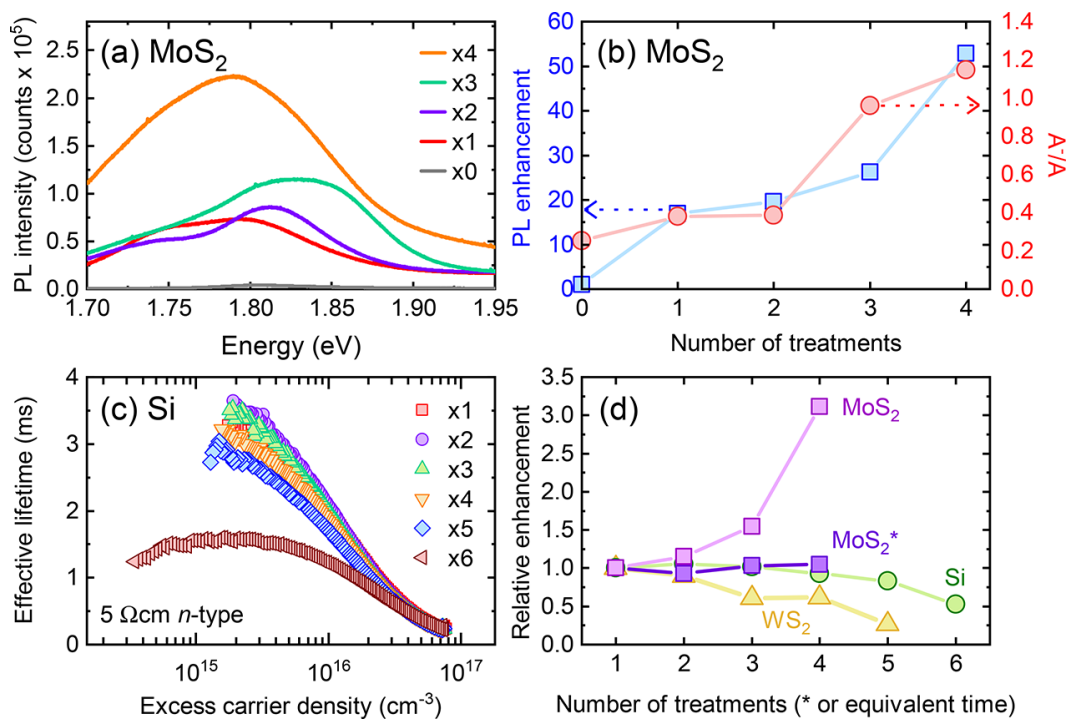

Figure 2. (a) PL spectra of $\mathrm{MoS}_{2}$ flakes on $\mathrm{SiO}_{2} / \mathrm{Si}$ following successive treatments in TFSA-DCM. The data for 1-4 treatments are from the same flake. (b). PL enhancement factor from the data in (a) on the left axis with the ratio of the PL intensity for the trion ( $\mathrm{A}^{-}$) to that of the A exciton on the right axis. (c) Effective lifetime of a single silicon sample $(5 \Omega \mathrm{cm}, n$-type, $\sim 125 \mu \mathrm{m}$ thick) successively treated with TFSADCM. (d) Change in performance relative to the first treatment in TFSA-DCM using peak PL data for MoS $\mathrm{S}_{2}$ and WS $\mathrm{Wn}_{2}$ and effective lifetime data at an excess carrier density of $5 \times 10^{15} \mathrm{~cm}^{-3}$ for silicon. The additional data set for $\mathrm{MoS}_{2}$ marked $*$ is for flakes subjected to a single treatment in TFSA-DCM for the equivalent time duration of the multiple treatments.

results demonstrate that room temperature treatments with TFSA-DCM can result in good results while avoiding the safety risks of heating TFSA-DCE to $\sim 100{ }^{\circ} \mathrm{C}$, as done previously. ${ }^{3-5,11,12}$

The PL signals for untreated few-layer and monolayer $\mathrm{MoS}_{2}$ were centered at $\sim 1.86 \mathrm{eV}$ (Figure 1c) and $\sim 1.87 \mathrm{eV}$ (Figure 1d), respectively, consistent with literature reports for untreated few-layer $^{38}$ and monolayer ${ }^{5,13,32,38}$ samples. Our main untreated $\mathrm{WS}_{2} \mathrm{PL}$ signal was centered at $\sim 1.94 \mathrm{eV}$ (Figure 1g). PL spectra for untreated monolayer $\mathrm{WS}_{2}$ can vary quite widely with peak emissions in the approximate range 1.92-2.01 eV, depending on the sample production conditions. $^{39-41}$ Our peak lies in this range, and its energy is in agreement with other studies on exfoliated $\mathrm{WS}_{2}$. ${ }^{35,39,42}$

The enhancement factor (defined as $I_{\text {treated }} / I_{\text {control }}$ where $I_{\text {treated }}$ and $I_{\text {control }}$ are the maximum PL intensities of the treated and control samples, respectively) following room temperature treatment with TFSA-DCM was $\sim 18$ for $\mathrm{MoS}_{2}$ flakes, $\sim 43$ for monolayer $\mathrm{MoS}_{2}$, and $\sim 3$ for $\mathrm{WS}_{2}$ flakes. Although these enhancements are less pronounced than in some reports, ${ }^{3,5,36}$ they are comparable with other elevated temperature studies, ${ }^{7,10-12}$ demonstrating the promise of room temperature treatments.

The reasons for the solvent dependence are not yet clear. Spectra from ${ }^{19} \mathrm{~F}\left\{{ }^{1} \mathrm{H}\right\}$ NMR of the three solutions studied in Figure $1 \mathrm{~g}$ show a single peak at -76.7 or $-76.6 \mathrm{ppm}$. These shifts are the same within measurement variation (typically $\leq 1$ $\mathrm{ppm})$ and are consistent with previous TFSA studies. ${ }^{17,43}$ There is therefore no detectable difference in the fluorine environment in the different solutions nor is there any evidence for the TFSA dissociating (at all, or differently in the different solvents) in solution, prior to interacting with the surface. Specifically, no peak at $-80 \mathrm{ppm}$ was detected, which is believed to occur when TFSA breaks down. ${ }^{17}$

Previous work for silicon found that the relative polarity of the solvent had no obvious bearing on the TFSA passivation quality, ${ }^{16,18}$ and this is the case for $\mathrm{MoS}_{2}$ and $\mathrm{WS}_{2}$ also. Data in
Figure $1 \mathrm{c}, \mathrm{d}, \mathrm{g}$ show that the nonpolar solvent (pentane) gives substantially better results than DCE (relative polarity of $0.327)^{44}$ for $\mathrm{WS}_{2}$, whereas DCM, whose relative polarity of $0.309^{44}$ is only just less than that of DCE, offers the best outcome for both $\mathrm{MoS}_{2}$ and $\mathrm{WS}_{2}$. Understanding this solvent dependency is the subject of further study.

We next address the issue of the number of processing steps required to achieve optimal performance. Early reports of superacid-based $\mathrm{MoS}_{2}$ treatments claim that, to achieve high enhancement levels, it is sometimes necessary to repeat the procedure several times; ${ }^{3}$ however we are not aware that such procedures have been applied to either silicon or mechanically exfoliated $\mathrm{WS}_{2}$. Figure 2 presents data for multiple TFSADCM treatments on $\mathrm{MoS}_{2}, \mathrm{WS}_{2}$, and silicon with the same TMDC flake or silicon wafer sample measured after each treatment. For $\mathrm{MoS}_{2}$ in Figure 2a, we find the PL intensity increases with the number of treatments. The enhancement factor after four immersions in TFSA-DCM is $\sim 53$, which is larger than many elevated temperature studies. ${ }^{7,9,10,12}$ After four treatments, the $\mathrm{MoS}_{2}$ flake under investigation detached from the substrate and was lost.

The energy dependence of the $\mathrm{MoS}_{2} \mathrm{PL}$ spectra in Figure 2a changes with the number of treatments. $\mathrm{MoS}_{2} \mathrm{PL}$ is believed to comprise trion $\left(\mathrm{A}^{-}, \sim 1.81 \mathrm{eV}\right)$ and two exciton $(\mathrm{A}, \sim 1.86 \mathrm{eV}$ and $\mathrm{B}, \sim 1.99 \mathrm{eV})$ emissions, which can be fitted with Lorentzian peaks. ${ }^{12}$ We provide peak deconvolution information for $\mathrm{MoS}_{2}$ in Figure S2. Monitoring evolution of the A and $\mathrm{A}^{-}$peaks shows an increase in $\mathrm{A}^{-} / \mathrm{A}$ ratio with increasing $\mathrm{PL}$, as shown in Figure $2 b$.

We find that the PL emission energy peak from $\mathrm{MoS}_{2}$ can shift slightly in response to a TFSA-based treatment with a small red shift found for once-treated few-layer flakes in Figure 1c, a small blue shift found for once-treated CVD monolayers in Figure 1d, and shifts in both directions found after multiple treatments for few-layer flakes in Figure 2a. Studies on TFSAtreated flakes have reported either essentially no shift ${ }^{3,6,45-47}$ or a blue shift, ${ }^{13,37}$ but all these studies used monolayers 


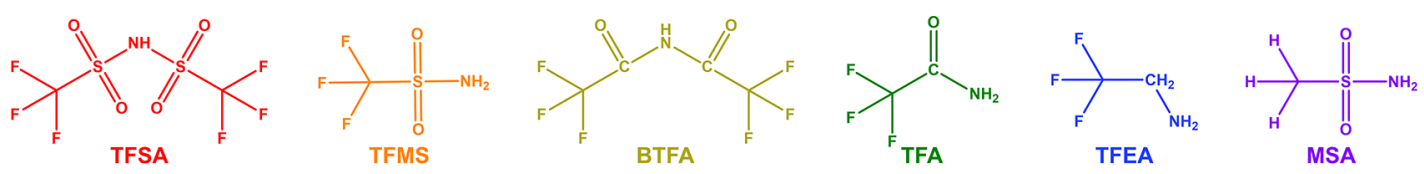

$\square$ TFSA $\bigcirc$ TFMS $\triangle$ BTFA $\nabla$ TFA $\diamond$ TFEA $\triangleleft$ MSA
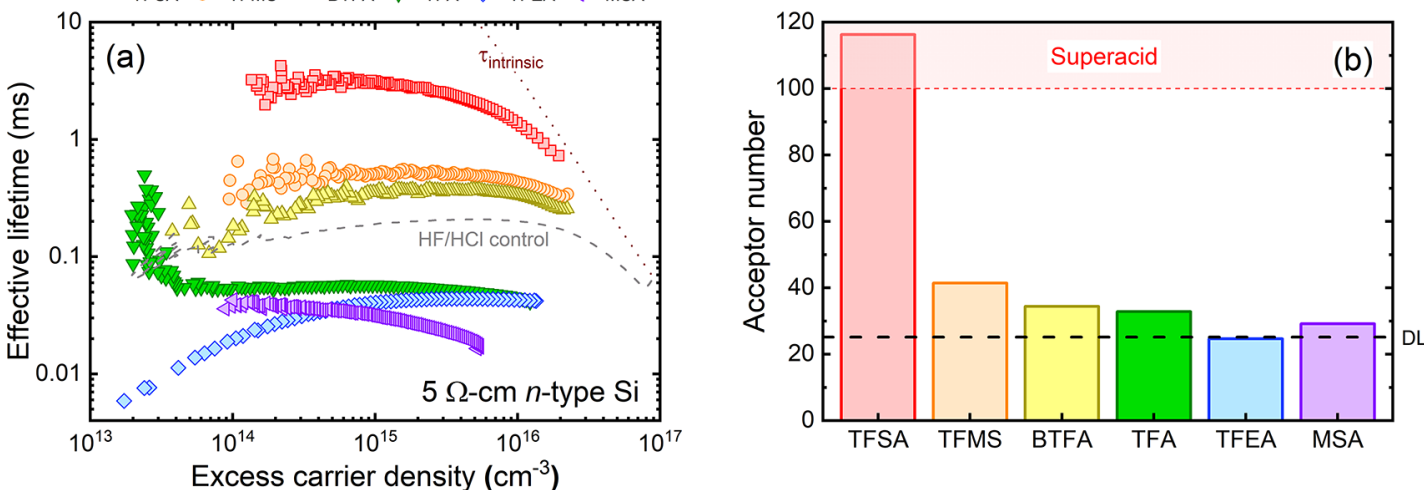

Figure 3. Top: Schematic chemical structures for analogue species investigated. Bottom: (a) Excess carrier density versus effective lifetime for $\sim 125 \mu \mathrm{m}$ thick $5 \Omega \mathrm{cm} n$-type silicon samples passivated with different species in DCM. Also shown is the intrinsic lifetime limit ${ }^{51}$ as a dotted curve with experimental data for a $\mathrm{HF} / \mathrm{HCl}$ treated control, additionally shown as a dashed curve. (b) Acceptor numbers for analogue species determined by the Gutmann-Beckett method. The lower dashed line corresponds to TPO (25.2) to give the detection limit (DL). ${ }^{18}$ Species exceeding the upper dashed line at 100 are Lewis superacidic. ${ }^{52}$

whereas our flakes were a few layers thick and so may behave differently. Studies on TFSA-treated CVD monolayers have observed blue shifts, ${ }^{9,10,12,48}$ consistent with our observations. One multiple treatment study on a monolayer flake showed an initial blue shift relative to the untreated case followed by red shifts with successive treatments. ${ }^{11}$ Thus, it is clear that small shifts in either direction are possible, dependent on the finer details of the samples studied.

Interestingly, both silicon and $\mathrm{WS}_{2}$ show different behaviors to $\mathrm{MoS}_{2}$ when the same samples are subjected to multiple TFSA-DCM treatments. For silicon, the effective lifetime reduces with multiple treatments, as shown in Figure 2c. For $\mathrm{WS}_{2}$, the PL signal from a given flake also reduces, as shown in Figure S3. Figure $2 \mathrm{~d}$ summarizes the relative changes. The difference between the $\mathrm{MoS}_{2}$ and $\mathrm{WS}_{2}$ flakes suggests that the effect is material specific and not general to TMDCs. A recent report on CVD $\mathrm{WS}_{2}$ monolayers reported an increase in PL intensity following successive treatments, ${ }^{49}$ but in this report, enhancement was achieved via drop-casting the solution onto the surface rather than treating via immersion, as was the case here. This further emphasizes the difficulty in understanding TMDC enhancement treatments, as differences in sample condition and processing mean enhancements differ considerably between reports. As TFSA is reported to physisorb onto $\mathrm{WS}_{2}$ and is removed by rinsing with solvent, ${ }^{49}$ it is not surprising that reimmersion of $\mathrm{WS}_{2}$ flakes into solution could cause the enhancement effects to be lost. Additionally, for silicon, it has been proposed that TFSA (or its constituents) passivate silicon through forming a protective hydrophobic barrier at the surface. ${ }^{14,16}$ If this were the case, then reimmersion into solution could cause this protective barrier to redissolve into its original solution, as is the case for $\mathrm{WS}_{2}{ }^{49}$

As the $\mathrm{MoS}_{2}$ flake from which Figure 2a data were obtained was known to be a few layers thick, the increase in PL might have arisen from exfoliation of the top layers with each immersion in solution, resulting in successively fewer layers and consequently greater PL intensity. ${ }^{33}$ However, Raman spectra collected following each treatment indicated no decrease in layer thickness. Furthermore, as reports on repeat treatments focus on $\mathrm{MoS}_{2}$ monolayers, ${ }^{3,11}$ the behavior observed here is consistent with a surface effect, rather than the effect of studying a few-layer sample. It is also clear that this increase is due to the repeat treatments, rather than simply a result of increasing treatment time, as data for flakes immersed in TFSA-DCM for equivalent time durations (marked with an asterisk in Figure 2d) do not show a significant change in PL. Similar data for other materials are provided in Figure S4.

A further difference between $\mathrm{MoS}_{2}$ and silicon is the longevity of the superacid treatment. In both cases, TFSAbased benefits are said to be temporary, with silicon passivation degrading quickly under ambient conditions, while $\mathrm{MoS}_{2}$ enhancement, though considerably more long-lived, ${ }^{3,16}$ also degrades over extended periods. ${ }^{46}$ The stability of the room temperature treatment of $\mathrm{MoS}_{2}$ was determined through measurements over the course of 10 days under ambient conditions, as shown in Figure S5 alongside equivalent data for silicon. Over the course of 10 days, the PL intensity of treated $\mathrm{MoS}_{2}$ decreased slightly, although the enhancement following ambient exposure remained considerably higher than some high-temperature passivation reports. ${ }^{7,9,10}$ Despite the degradation, the stability of the room temperature process seems broadly comparable to that of the high-temperature processes. ${ }^{3}$

Until this stage, we have focused on effects arising from a TFSA-based solution. While this provides property enhancement of all three materials studied, the mechanism by which this occurs is unclear. It is not clear what specific functional group(s) from the TFSA molecule give(s) rise to the effects observed. To gain insight into this, five chemical analoguesstructurally related to TFSA but differing in key functionality-were considered: trifluoromethanesulfonamide (TFMS), bistrifluoroacetamide (BTFA), 2,2,2-trifluoroacetamide (TFA), 2,2,2-trifluoroethylamine (TFEA), and methanesulfonamide (MSA). These are shown schematically at the top of Figure 3. TFMS, a synthetic precursor to TFSA, ${ }^{43}$ corresponds to one moiety of the symmetrical TFSA species and is thought to be a potential TFSA dissociation product. ${ }^{22}$ BTFA, like TFSA, comprises two symmetrical units around a 
Table 1. Effective Lifetime and Upper-Limit Surface Recombination Velocity $(S)$ at an Excess Carrier Density of $10^{15} \mathrm{~cm}^{-3}$ for Silicon Wafers Treated with Analogue Solutions in $\mathrm{DCM}^{a}$

$\begin{array}{llccccc} & \text { TFSA } & \text { TFMS } & \text { BTFA } & \text { TFA } & \text { TFEA } & \text { MSA } \\ \text { effective lifetime (ms) } & 3.06 & 0.51 & 0.35 & 0.057 & 0.040 & 0.031 \\ \text { upper-limit } S(\mathrm{~cm} / \mathrm{s}) & 1.9 & 12.1 & 17.9 & 109 & 154 & 200 \\ \text { acceptor number } & 116.3^{18} & 41.4 & 34.4 & 32.8 & 24.7 & 29.2\end{array}$

${ }^{a}$ Also stated is the acceptor number for each passivating species, which was determined using Equation 2.
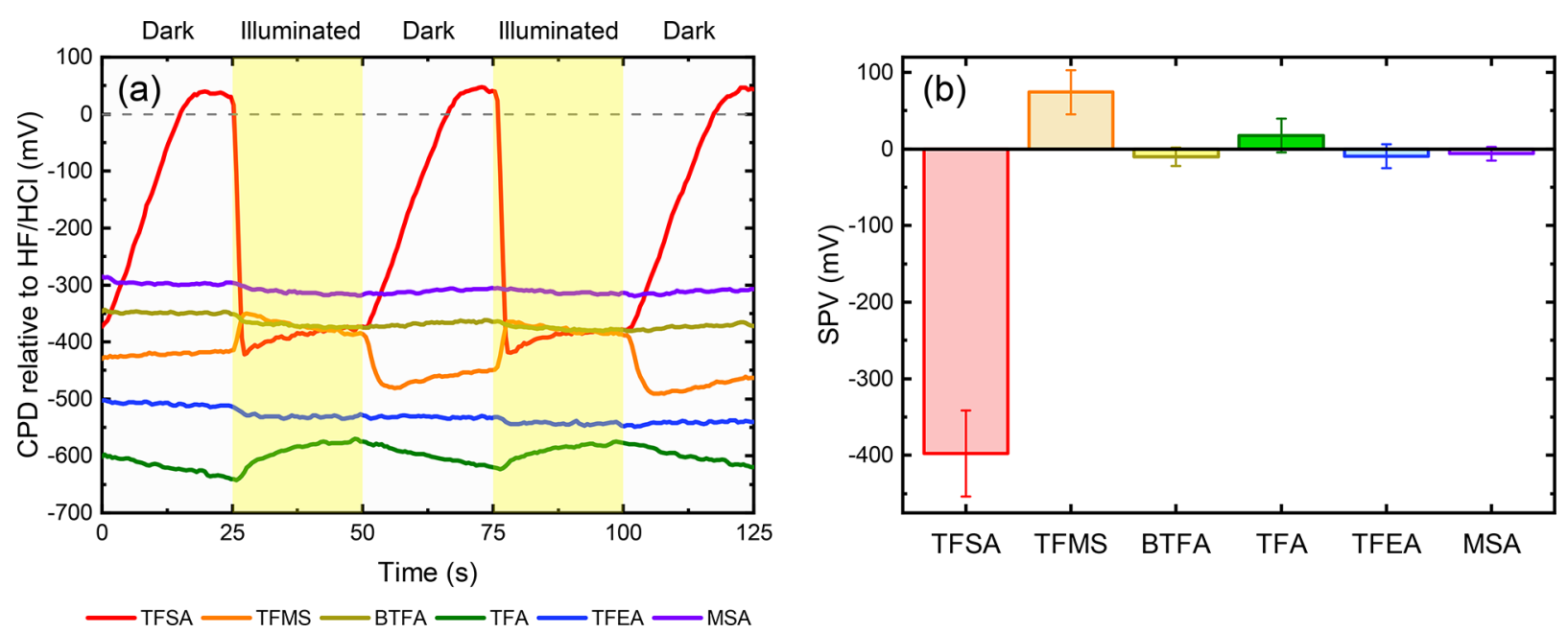

Figure 4. Kelvin probe data for $5 \Omega \mathrm{cm} n$-type silicon wafers treated with analogue solutions in DCM. (a) Contact potential difference (CPD) relative to $\mathrm{HF} / \mathrm{HCl}$-dipped sample. (b) Surface photovoltage (SPV) defined as CPD measured under illumination minus the CPD measured in the dark.

central amide, but the sulfonyl groups are substituted for carbonyl groups. The other three species-TFA, TFEA, and MSA - are similar to TFMS but with various substitutions. TFA corresponds to one moiety of BTFA, while in TFEA the sulfonyl functionality is replaced with an alkyl group. MSA retains the sulfonyl functionality but instead the $\mathrm{CF}_{3}$ group is substituted for $\mathrm{CH}_{3}$.

To assess the performance of the TFSA analogues, we first studied their effects on high-purity crystalline silicon samples. Silicon provides a well-controlled surface that can be controlled at the atomic level by wet chemical processing and characterized by carrier lifetime measurements of large area $\left(>1 \mathrm{~cm}^{2}\right)$ samples. ${ }^{50}$ We chose DCM as a common solvent as all analogues are soluble in it and because of its superiority in TMDC enhancement. Effective lifetime data for samples treated with each analogue species can be seen in Figure 3a. The performance of the solutions should be considered relative to the control sample subjected to just an $\mathrm{HF}-\mathrm{HCl}$ final cleaning step, as this last stage results in passivation, presumably due to hydrogen surface termination. ${ }^{50}$ TFSA, TFMS, and BTFA outperform the control, whereas TFA, TFEA, and MSA result in substantially less passivation. TFSA's passivating ability of silicon is far superior to that of all other species considered and gives lifetimes close to the intrinsic limit at high excess carrier densities. ${ }^{51}$

The passivation level can be quantified in terms of a surface recombination velocity, $S$, defined according to

$$
S=\frac{W}{2}\left(\frac{1}{\tau_{\text {effective }}}-\frac{1}{\tau_{\text {bulk }}}\right)
$$

where $W$ is the sample thickness and $\tau_{\text {effective }}$ and $\tau_{\text {bulk }}$ are the effective and bulk carrier lifetimes, respectively. The bulk lifetime is determined by intrinsic (i.e., radiative and Auger) and extrinsic recombination. We quantify the former using the parametrization of Niewelt et al., and the latter is difficult to quantify accurately, so we take it to be zero, which means our values of $S$ are upper limits. Such values extracted at an excess carrier density of $10^{15} \mathrm{~cm}^{-3}$ are given in Table 1 . TFSA gives $S$ $<2 \mathrm{~cm} / \mathrm{s}$, which is consistent with its performance with other solvents. $^{15,16,18,19}$

Species with similar structures to TFSA result in vastly different passivation behaviors when applied to silicon, as shown in Figure 3a. The solution's acidity could play a role in the passivation process, and acceptor numbers for the six compounds are shown in Figure $3 \mathrm{~b}$ and Table 1. These are calculated using Equation 2 on the basis of NMR data in Figure S6.

Only TFSA exhibits Lewis superacidity (since it has an acceptor number $>100)^{52}$ with TFSA's analogues being considerably less acidic. We note that previous work for silicon has demonstrated that bis(trifluoromethanesulfonyl)methane (TFSM) has an acceptor number of 70.7 and gives $S$ $<2 \mathrm{~cm} / \mathrm{s}$ in hexane, which demonstrates that high passivation levels do not require superacidity, ${ }^{18}$ although TFSM has a higher Lewis acidity than the analogues considered here. With the exception of MSA (whose dependence on excess carrier density is different), the effective lifetimes at $10^{15} \mathrm{~cm}^{-3}$ injection come in order of the acceptor number. A Nafion passivation study of silicon has also found a correlation between Lewis acidity and effective lifetime. ${ }^{53}$ It is however clear that the chemical's acidity is not the only factor in the level of passivation achieved. For example, BTFA and TFA have very similar acceptor numbers, yet their effect on the silicon surface is very different, with the former improving the 

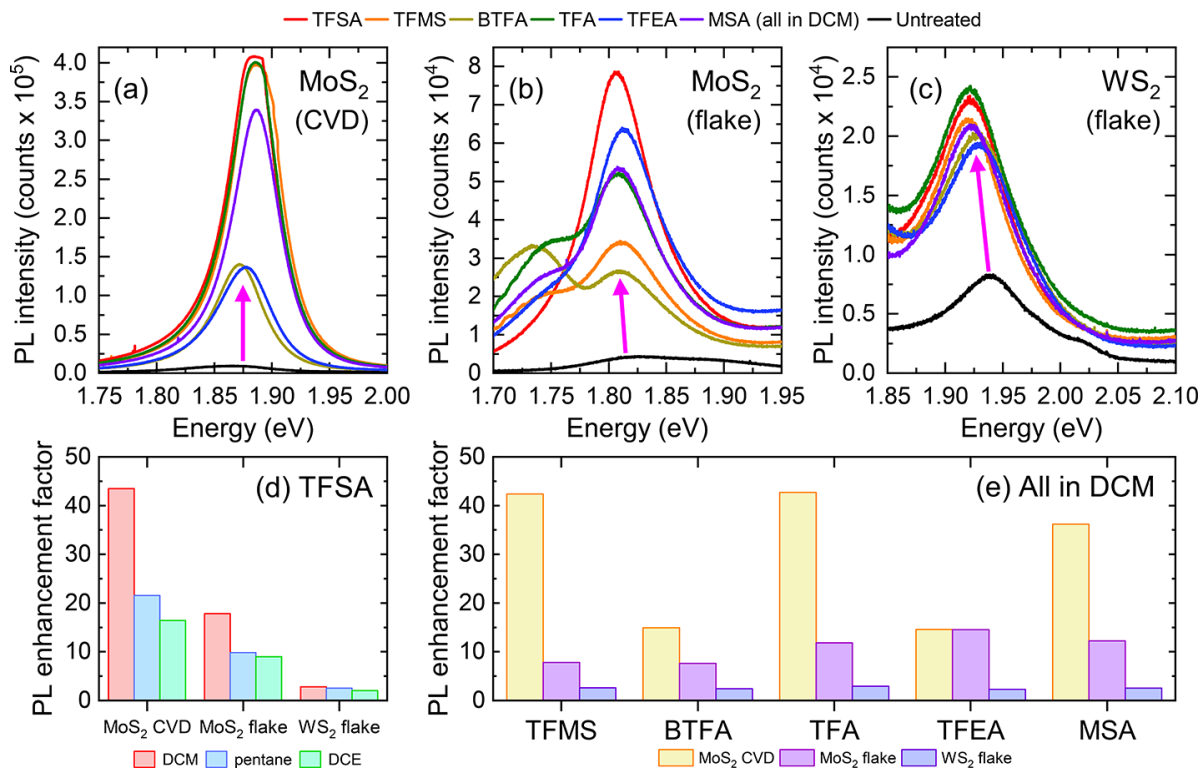

Figure 5. PL spectra for (a) CVD $\mathrm{MoS}_{2}$, (b) $\mathrm{MoS}_{2}$ flakes, and (c) $\mathrm{WS}_{2}$ flakes single-treated with solutions of TFSA and its analogues in DCM compared to untreated controls. (d) PL enhancement factors for $\mathrm{MoS}_{2}$ and WS treated once with solutions made with TFSA in the solvents stated. (e) PL enhancement factors for $\mathrm{MoS}_{2}$ and $\mathrm{WS}_{2}$ treated with solutions of TFSA analogues in DCM.

hydrogen-terminated control and the latter making it considerably worse.

In general, surface passivation of bulk semiconductors comprises both chemical and field-effect components. Both components are important for TFSA applied to silicon, with field-effect passivation arising from negative charge. ${ }^{17}$ The partial vacuum instability of the treatment is consistent with a charged thin film formed by physisorption on the surface supplementing the chemical passivation from hydrogen termination. ${ }^{17}$ Kelvin probe (KP) experiments were performed on $n$-type silicon samples treated with solutions in DCM of TFSA and its analogues. Figure $4 \mathrm{a}$ shows the contact potential difference (CPD) relative to a $\mathrm{HF} / \mathrm{HCl}$-dipped sample measured under a cycle of dark and light conditions.

Figure $4 \mathrm{~b}$ shows the surface photovoltage (SPV), which is the CPD measured under illumination minus the CPD measured in the dark. Previous work showed that the illumination of TFSA-hexane-treated silicon resulted in an increase in surface potential due to a reduction in the width of the depletion region. ${ }^{17}$ The CPD results here for silicon treated with TFSA-DCM are consistent with this, showing that a negative charge is also present with a different solvent here. On moving from illumination to dark conditions, the CPD for the TFSA-DCM case increases gradually over the next $\sim 20 \mathrm{~s}$. Such a slow recovery is attributed to trapped charges. ${ }^{54}$ Silicon passivated by TFSA analogue solutions show much smaller changes in CPD from dark to illuminated conditions than TFSA itself, and in some cases, the polarity of the change is different. As summarized by Figure 4b, TFMS-treated surfaces had a notable illumination response, but in contrast with TFSA, the CPD of TFMS-treated surfaces becomes less negative under illumination. This corresponds to a widening of the depletion region, which would be expected for a positively charged passivating film. ${ }^{55}$

The magnitude of the CPD difference for TFMS-treated surfaces is smaller than that for TFSA-treated surfaces, implying a lower charge density. Quantifying the level of charge in a film from a $\mathrm{KP}$ measurement alone is extremely difficult without more detailed knowledge of the film's structure. The slow emptying of states observed is also observed for TFMS but on a much shorter time scale. Of the other treatments, only the TFA-treated surface had a significant light response in the $\mathrm{KP}$ experiments, although it was much smaller than those observed for TFSA and TFMS, suggesting an even smaller field-effect contribution probably from a relatively low level of positive charge.

Like TFMS, the CPD for TFA increases on illumination, although this process is more gradual than for TFMS. Comparing the KP data in Figure 4 to the lifetime data and molecular structures in Figure 3 yields important insights. TFSA and TFMS treatments give the longest lifetimes (lowest $S$ ), and this is likely due to enhanced field-effect passivation due to higher levels of charge than for the other treatments. BTFA treatments result in good lifetimes, but the absence of significant field effect passivation means that the values are lower than for TFSA and TFMS. The lifetimes with BTFA are however higher than the hydrogen-terminated control, implying that the treatment results in a small enhancement in chemical passivation. The other three treatments (TFA, TFEA, and MSA) give rise to worse passivation than the HF/ HCl-treated control, with an absence of significant field effect passivation and a reduction in chemical passivation relative to a hydrogen-terminated surface.

The $\mathrm{KP}$ part of the study suggests that $\mathrm{CF}_{3} \mathrm{SO}_{2}$ functionality (present in TFSA and TFMS) appears to be required to form a charged film necessary to provide field-effect passivation. Replacing the sulfur for carbon (BTFA versus TFSA) greatly reduces the charge in the film. Replacing fluorine with hydrogen (MSA versus TFMS) also results in a relatively uncharged film. Attempts to characterize the films directly by techniques such as X-ray photoelectron spectroscopy are not possible as the films are not vacuum stable on silicon. ${ }^{17}$

We now turn our attention to the treatment of TMDC materials with TFSA analogue solutions in DCM, with results from PL experiments on single-treated samples presented in Figure 5 alongside those from untreated controls. All the 
treatments used enhance the PL intensity relative to the control for both $\mathrm{MoS}_{2}$ and $\mathrm{WS}_{2}$, shown in Figure $5 \mathrm{a}-\mathrm{c}$, respectively. Peak fitting for $\mathrm{MoS}_{2}$ and $\mathrm{WS}_{2}$ flakes is shown in Figures S7 and S8, respectively. The enhancement factors are shown in Figure 5e, with the enhancement factors for TFSA in other solvents from Figure 1 also shown in Figure 5d. For monolayer $\mathrm{MoS}_{2}$, TFSA, TFMS, and TFA perform equally well, with enhancement factors of $\sim 43$. BTFA and TFEA perform the worst although still achieve an enhancement factor of $\sim 14$. For few-layer $\mathrm{MoS}_{2}$ flakes, TFSA-DCM gives an enhancement factor of $\sim 18$, with TFEA, MSA, and TFA also performing relatively well. The PL enhancements from $\mathrm{MoS}_{2}$ achieved with BTFA and TFMS are the lowest of our treatments, although even these enhancements exceed some reports for TFSA passivation. ${ }^{11,12}$ For $\mathrm{WS}_{2}$, the enhancement factors are fairly similar for TFSA and its analogues, in the range $\sim 2.2-2.9$.

The PL peak shapes for all treated monolayer $\mathrm{MoS}_{2}$ samples and $\mathrm{WS}_{2}$ flakes were very similar. In comparison, the shapes of the $\mathrm{MoS}_{2}$ few-layer flake PL spectra depend on the treatment applied. PL spectra from TMDC crystals can vary due to surface roughness, charge, defect concentration, and substraterelated effects. ${ }^{23,56}$ Broader peaks correspond to relatively higher electron densities and prominent $\mathrm{A}^{-}$emission shoulders, notable in the spectra of TFMS-, BTFA-, TFA-, and MSA-treated few-layer $\mathrm{MoS}_{2}$, and are likely due to charge accumulation at defect sites. ${ }^{10}$ In Figure S8, we fit the $\mathrm{WS}_{2} \mathrm{PL}$ spectra using two Lorentzian peaks, with the higher energy peak probably arising from the neutral A-exciton and the lower energy peak likely due to the trion state. ${ }^{35,39,57,58}$ The relatively low energy of the lower peak could also be consistent with defect-bound exciton emission. ${ }^{39,57,59}$

Previous $\mathrm{MoS}_{2}$ studies claim that Brønsted acidity is crucial, $^{7,24}$ with recent computational studies suggesting that enhancements arise due to the labile proton of TFSA occupying sulfur vacancies. ${ }^{24}$ However, our data show that acidity has no major influence, with there being no clear link between acceptor number in Figure $3 \mathrm{~b}$ and performance in Figure 5. TFA, TFEA, and MSA have very low acidity (with TFEA being completely nonacidic) and yet all three enhance PL considerably, in either in the few-layer sample, the monolayer sample, or both.

Although a high Brønsted acidity was thought necessary for effective treatments of $\mathrm{MoS}_{2}{ }^{7,24} \mathrm{WS}_{2}$ has previously been enhanced by treatment with oleic acid, a weak acid reported to be less acidic than the species discussed here. ${ }^{36,60}$ The nonacidic analogues here enhance $\mathrm{WS}_{2}$ with similar effectiveness to TFSA, further suggesting that acidity is not a prerequisite for effective $\mathrm{WS}_{2}$ treatment. Additionally, oleic acid lacks sulfonyl functionality, suggesting that the mechanisms proposed for $\mathrm{MoS}_{2}$ enhancements are not necessarily applicable to $\mathrm{WS}_{2}$.

Computational studies claim that TFSA enhances $\mathrm{MoS}_{2}$ via dissociation in situ, releasing $\mathrm{SO}_{2}$, which either "repairs" sulfur defects or fills vacancies with free oxygen or sulfur, ",9 although a recent report claims that TFSA creates/opens sulfur defects through the removal of oxygen. ${ }^{37}$ As the results here show that the quality achieved by treating $\mathrm{MoS}_{2}$ with TFSA is comparable to that achieved with TFA (which does not contain a sulfonyl group) and, in the case of few-layer $\mathrm{MoS}_{2}$, TFEA (which contains neither sulfur nor oxygen), it does not seem likely that this proposed mechanism can explain the behavior observed in all cases. Additionally, NMR experiments
(Figure S9) show no evidence of dissociation of TFSA or its analogues in solution, prior to TFSA interacting with the surface. Our NMR experiments do not provide information on any molecular dissociation that may occur upon interaction with the TMDC surface however.

There are also computational reports that claim that TFSAbased treatment of $\mathrm{WS}_{2}$ involves "repairing" sulfur vacancies by $\mathrm{SO}_{2} .{ }^{9}$ Data in Figure 5 show that analogues to TFSA behave very similarly to TFSA itself. Again, TFA and TFEA do not contain sulfonyl groups yet enhance $\mathrm{WS}_{2}$ as effectively as TFSA, thus suggesting that the presence of a sulfonyl group is not required for effective treatment of $\mathrm{WS}_{2}$. Alternative mechanisms to sulfur vacancy passivation need to be considered to explain TFSA analogue enhancement of TMDCs. Other chemical treatments of TMDCs have been reported using species without sulfonyl functionality, ${ }^{36,61}$ but this work focuses on understanding the behavior of TFSA rather than finding alternative treatment families.

When comparing the relative effects of the treatments on silicon and the TMDC materials, it is important to note the initial reference states differ considerably. It is well-known how to functionalize a silicon surface by applying a $\mathrm{HF} / \mathrm{HCl}$ pretreatment to hydrogen-terminate the surface, ${ }^{50}$ but for TMDCs, the starting condition is less well-defined. Thus, the degradation of silicon from certain analogue treatments occurs relative to an already good starting condition, whereas the enhancement of TMDCs by all analogue treatments could be a reflection of their less well-understood surface preparation. For silicon, there is a field effect passivation component trend as shown by the KP data in Figure 4. The same trends do not hold for all the TMDC materials studied. For monolayer $\mathrm{MoS}_{2}$, TFSA-, TFMS-, and TFA-based solutions, the only ones with a notable light response on silicon, give rise to the greatest enhancements. This suggests that charge transfer to the $\mathrm{MoS}_{2}$ film could be further investigated as a possible mechanism. For $\mathrm{WS}_{2}$, all the treatments give similar enhancement factors, despite giving rise to differing levels of charge on silicon, suggesting a different microscopic mechanism than for $\mathrm{MoS}_{2}$. Additionally, for few-layer $\mathrm{MoS}_{2}$, good results are achieved with TFSA, but TFMS is a relatively weak performer despite being positively charged when on silicon.

In summary, while TFSA and its analogues can enhance the properties of silicon, CVD monolayer $\mathrm{MoS}_{2}$, and $\mathrm{MoS}_{2} / \mathrm{WS}_{2}$ flakes, the mechanisms by which this arises may not have a great deal in common. Modern silicon wafers have close to zero bulk recombination, ${ }^{51}$ so the effective lifetime is strongly influenced by the surfaces. The excellent surface passivation of silicon by TFSA is a consequence of both chemical and field effect passivation. The TMDC materials are only a monolayer or several monolayers thick and are likely to contain relatively high levels of defects. It is not clear from our study whether the TFSA family treatments are affecting the surfaces or bulk defects.

Our work has shown that there is still a lot to understand regarding TFSA-based treatments of $\mathrm{MoS}_{2}$ and $\mathrm{WS}_{2}$, with acidity and the inclusion of sulfur and oxygen from TFSA not necessarily playing the roles previously suggested. Developing a deeper understanding of the interactions of TFSA is of relevance to other fields, including other $2 \mathrm{D}$ materials or batteries, where TFSA-based electrolytes appear promising due to their compatibility with anode materials. ${ }^{22,62,63}$ 


\section{CONCLUSIONS}

The performance of $\mathrm{MoS}_{2}, \mathrm{WS}_{2}$, and silicon following treatment by TFSA and a series of analogue species with specific compositional differences has been studied experimentally. We find considerable enhancements at room temperature when DCM is used as the solvent, with monolayer $\mathrm{MoS}_{2}$ enhanced by $\sim 43$ following a single treatment with TFSA-DCM, and an enhancement of $\sim 53$ achievable for fewlayer $\mathrm{MoS}_{2}$ following multiple treatments. Our room temperature approach is more practical than previous studies that use a more hazardous solvent (DCE) at elevated temperatures $\left(\sim 100{ }^{\circ} \mathrm{C}\right)$.

Surface passivation by solutions analogous to TFSA was first studied for silicon, with TFMS and BTFA outperforming hydrogen-terminated controls without them being Lewis superacids. $\mathrm{KP}$ experiments confirm the presence of a charged film when the chemical possesses a $\mathrm{CF}_{3} \mathrm{SO}_{2}$ functional group, with the film being negatively charged for TFSA and positively charged for TFMS. Treatments with the analogue solutions were also applied to $\mathrm{MoS}_{2}$ and $\mathrm{WS}_{2}$, with all treatments enhancing PL relative to untreated controls regardless of the acidity level of the solution. Trends from silicon surface passivation were not generally replicated for the TMDCs, suggesting the mechanism of enhancement is quite different. Both $\mathrm{MoS}_{2}$ and $\mathrm{WS}_{2}$ can be enhanced with analogues without sulfonyl groups, sulfur, or oxygen, meaning alternatives to the theoretically proposed sulfur vacancy occupation mechanism must be considered.

\section{METHODS}

Chemicals and Materials. 1,2-Dichloroethane (DCE, $\geq 99.8 \%$ ), bis(trifluoroacetamide) (BTFA), bis(trifluoromethane)sulfonamide (TFSA, $\geq 95.0 \%)$, pentane ( $>99 \%)$, triethylphosphine oxide (TPO, 97\%), trifluoromethanesulfonamide (TFMS, 95\%), and $\alpha, \alpha, \alpha$ trifluorotoluene $(\geq 99 \%)$ were obtained from Sigma-Aldrich. 2,2,2Trifluoroacetamide (TFA, 97\%), dichloromethane (DCM, 99.7+\%), and methanesulfonamide (MSA, 98+\%) were obtained from AlfaAesar. Deuterated chloroform ( $>99.8 \%$ purity with $0.03 \%$ tetramethylsilane) was obtained from Apollo Scientific, and 2,2,2-trifluoroethylamine (TFEA) was obtained from Fisher Scientific. $\mathrm{MoS}_{2}$ and $\mathrm{WS}_{2}$ crystals $(\geq 99.999 \%)$ grown by chemical vapor transport (CVT) were obtained from Ossila, as was a $\mathrm{MoS}_{2} \mathrm{CVD}$ monolayer film (>99\%) on $\mathrm{SiO}_{2} / \mathrm{Si}$. Isopropanol and acetone, used for cleaning TMDC substrates, were cleanroom grade. Substrates for lifetime and Kelvin probe measurements were high quality, $\sim 125 \mu \mathrm{m}$ thick, (100) orientation, $5 \Omega \mathrm{cm}$, unpolished $n$-type Czochralski silicon wafers. Lifetime measurements were made on $5 \times 5 \mathrm{~cm}^{2}$ samples, which were sufficiently large to avoid strong edge recombination effects. ${ }^{64}$ Substrates for TMDC exfoliation were polished $750 \mu \mathrm{m}$ thick silicon wafers coated with $325 \mathrm{~nm}$ of $\mathrm{SiO}_{2}$.

Silicon Passivation. Silicon wafers were prepared following a previously reported method, with the final preparation step (immersion in $2 \% \mathrm{HF}$ for $60 \mathrm{~s}$ ) modified to be immersion in $1 \%$ $\mathrm{HF} / 1 \% \mathrm{HCl}$ for $5 \mathrm{~min}$, as such solutions offer superior passivation quality. ${ }^{50}$ Passivation was also conducted following a previously reported room temperature method, whereby TFSA or its analogues were dissolved in anhydrous solvent at a ratio of $2 \mathrm{mg} / \mathrm{mL}{ }^{16}$ It is known that pentane-based solutions offer the best known passivation quality, ${ }^{18}$ but, as not all the species used here are soluble in pentane, DCM was chosen as a common solvent. TFSA, BTFA, and TFMS were handled in a high specification, filtered, sealed MBRAUN UNIlab modular glovebox with a gas purification system, solvent filter, and a controlled low-humidity atmosphere $\left(<0.1 \mathrm{ppm}\right.$ of $\mathrm{O}_{2}$ and $\mathrm{H}_{2} \mathrm{O}$ ). MSA, TFEA, and TFA were handled in a lower specification glovebox with ambient flowing nitrogen (relative humidity <25\%). All solutions were prepared in the lower specification glovebox and stored in sealed containers before use. Cleaned wafers were then submerged in the solution for $60 \mathrm{~s}$ at room temperature before being removed and allowed to dry. Passivated samples were then placed in plastic Petri dishes and removed from the glovebox for characterization.

Lifetime and Kelvin Probe Measurements. Photoconductance decay (PCD) lifetime measurements were performed at room temperature using a Sinton WCT-120 lifetime tester. Lifetime measurements are assumed to be accurate to $\pm 5 \%{ }^{65}$ Contact potential difference (CPD) measurements were made with a KP Technologies SKP5050 Kelvin probe with a gold-plated tip on the basis of the method of Baikie et al. ${ }^{66}$ A Fiber-Lite DC-950 quartz tungsten halide (QTH) lamp was used for surface photovoltage (SPV) measurements. The light intensity measured at the sample location with a Thorlabs PM16-130 power meter was $0.77 \mathrm{~W} / \mathrm{cm}^{2}$ at $635 \mathrm{~nm}$.

NMR and Gutmann-Beckett Acidity Measurements. The Gutmann-Beckett method quantifies Lewis acidity by utilizing ${ }^{31} \mathrm{P}$ NMR shift of a reference compound (triethylphosphine oxide, TPO), which is highly sensitive to its environment to determine an "acceptor number" ${ }^{67,68}$ The Lewis basic oxygen in TPO interacts with Lewis acids, deshielding the phosphorus center. The resulting ${ }^{31} \mathrm{P}$ shift in parts per million, $\delta_{\text {peak }}$ can be used to quantify the acceptor number: ${ }^{68}$

$$
\mathrm{AN}=2.21 \times\left(\delta_{\text {peak }}-41.00\right)
$$

The analyte and TPO were dissolved in $\mathrm{CDCl}_{3}$ in the MBRAUN glovebox. ${ }^{31} \mathrm{P}\left\{{ }^{1} \mathrm{H}\right\}$ spectra were recorded on a Bruker Avance III HD 300 at $121.5 \mathrm{MHz}$. ${ }^{1} \mathrm{H}$ and ${ }^{19} \mathrm{~F}\left\{{ }^{1} \mathrm{H}\right\}$ spectra were collected at 300 and 282.4 MHz, respectively, with $\alpha, \alpha, \alpha$-trifluorotoluene as an internal fluorine reference. All spectra were averaged over 64 scans.

TMDC Treatments. $\mathrm{MoS}_{2}$ and $\mathrm{WS}_{2}$ flakes were transferred onto a $\mathrm{SiO}_{2} / \mathrm{Si}$ substrate through mechanical exfoliation, and substrates were then rinsed in acetone/isopropanol to remove tape residue. Acetone and isopropanol are reported to suppress $\mathrm{MoS}_{2} \mathrm{PL}$ intensity, although their use is standard in TMDC device fabrication. ${ }^{23}$ Control experiments (Figure S1) indicate that, in this case, any differences in resulting $\mathrm{PL}$ as a result of surface pretreatments are negligible following treatment with TFSA. The room temperature passivation procedure followed was the same as for silicon substrates, but the immersion time was extended to $10 \mathrm{~min}$, in line with similar studies. $3,5,7$ This time is not thought to be sufficient to affect the $\mathrm{SiO}_{2}$ layer to a significant degree. The passivating solutions used here were more concentrated than those used in some TMDC passivation studies, ${ }^{3,5,6}$ but our concentration was kept consistent with the solutions used for silicon passivation.

Raman and Photoluminescence Spectroscopy. Raman and PL experiments were performed at room temperature using a Renishaw inVia Reflex Raman microscope using a $532 \mathrm{~nm}$ excitation laser set to $5 \%$ of maximum power $(\sim 0.9 \mathrm{~mW})$. All data were collected in confocal mode using a $50 \times$ Leica objective lens with a numerical aperture of 0.75 and an 1800 lines $/ \mathrm{mm}$ grating. The Raman data for $\mathrm{MoS}_{2}$ and $\mathrm{WS}_{2}$ flakes were the sum of four accumulations of 4 $\mathrm{s}$ each, and the PL data were the sum of four accumulations of $10 \mathrm{~s}$ each. Control experiments, shown in Figure S10, were conducted to determine the optimum laser exposure conditions for monolayer $\mathrm{MoS}_{2}$. Unless otherwise stated, the Raman and PL data of monolayer $\mathrm{MoS}_{2}$ were collected at a laser intensity of $0.1 \%(18 \mu \mathrm{W})$ and the PL data were the sum of four accumulations of $5 \mathrm{~s}$ each.

Data were acquired with the Renishaw WiRE 3.1 software package. Cosmic-ray features were removed from spectral data as necessary. Suitable $\mathrm{MoS}_{2}$ and $\mathrm{WS}_{2}$ flakes were identified utilizing Raman spectroscopy, and layer thickness determined by focusing on the $\mathrm{E}_{2 \mathrm{~g}}^{1}$ and $\mathrm{A}_{1 \mathrm{~g}}$ vibration modes $\left(\sim 380-410 \mathrm{~cm}^{-1}\right.$ for $\mathrm{MoS}_{2}, \sim 360-410$ $\mathrm{cm}^{-1}$ for $\left.\mathrm{WS}_{2}\right)^{29,34}$

\section{ASSOCIATED CONTENT}

\section{(s) Supporting Information}

The Supporting Information is available free of charge at https://pubs.acs.org/doi/10.1021/acsnano.1c09085. 
Figures of PL spectra, peak fitting for PL spectra, comparison of performance following repeat treatments, ambient stability of TFSA-DCM, ${ }^{31} \mathrm{P}\left\{{ }^{1} \mathrm{H}\right\},{ }^{19} \mathrm{~F}\left\{{ }^{1} \mathrm{H}\right\}$, and ${ }^{1} \mathrm{H}$ NMR spectra, peak fitting of $\mathrm{PL}$ signal, and $\mathrm{PL}$ intensities (PDF)

\section{AUTHOR INFORMATION}

\section{Corresponding Authors}

Sophie L. Pain - School of Engineering, University of Warwick, Coventry CV4 7AL, United Kingdom; ○ orcid.org/0000-0003-1333-2023; Email: sophie.pain@ warwick.ac.uk

John D. Murphy - School of Engineering, University of

Warwick, Coventry CV4 7AL, United Kingdom;

다이.org/0000-0003-0993-5972;

Email: john.d.murphy@warwick.ac.uk

\section{Author}

Nicholas E. Grant - School of Engineering, University of Warwick, Coventry CV4 7AL, United Kingdom; (1) orcid.org/0000-0002-3943-838X

Complete contact information is available at: https://pubs.acs.org/10.1021/acsnano.1c09085

\section{Author Contributions}

All authors contributed to the principal objectives of the work, helped devise experimental plans, and interpreted data. S.L.P. performed the experimental work, including all sample processing and characterization. S.L.P. and J.D.M. produced the figures and wrote the manuscript jointly, which was commented upon by N.E.G..

\section{Funding}

S.L.P. acknowledges funding from the Engineering and Physical Sciences Research Council Doctoral Training Partnership (EP/R513374/1).

\section{Notes}

Data underpinning figures in this paper can be freely downloaded from http://wrap.warwick.ac.uk/160908/. Requests for additional data should be made directly to the corresponding authors.

The authors declare no competing financial interest.

\section{ACKNOWLEDGMENTS}

The authors acknowledge the help of Dr. Ben Breeze and the use of Raman/PL facilities within the Spectroscopy RTP at the University of Warwick and Dr. Ivan Prokes of the Department of Chemistry, University of Warwick, for access to NMR facilities and experimental support.

\section{REFERENCES}

(1) Lien, D. H.; Uddin, S. Z.; Yeh, M.; Amani, M.; Kim, H.; Ager, J. W., III; Yablonovitch, E.; Javey, A. Electrical Suppression of All Nonradiative Recombination Pathways in Monolayer Semiconductors. Science 2019, 364 (6439), 468-471.

(2) Kim, H.; Uddin, S. Z.; Higashitarumizu, N.; Rabani, E.; Javey, A. Inhibited Nonradiative Decay at All Exciton Densities in Monolayer Semiconductors. Science 2021, 373 (6553), 448-452.

(3) Amani, M.; Lien, D. H.; Kiriya, D.; Xiao, J.; Azcatl, A.; Noh, J.; Madhvapathy, S. R.; Addou, R.; Kc, S.; Dubey, M.; Cho, K.; Wallace, R. M.; Lee, S. C.; He, J. H.; Ager, J. W., III; Zhang, X.; Yablonovitch, E.; Javey, A. Near-Unity Photoluminescence Quantum Yield in $\mathrm{MoS}_{2}$. Science 2015, 350 (6264), 1065-1068.
(4) Amani, M.; Burke, R. A.; Ji, X.; Zhao, P.; Lien, D. H.; Taheri, P.; Ahn, G. H.; Kirya, D.; Ager, J. W., III; Yablonovitch, E.; Kong, J.; Dubey, M.; Javey, A. High Luminescence Efficiency in $\mathrm{MoS}_{2}$ Grown by Chemical Vapor Deposition. ACS Nano 2016, 10 (7), 6535-6541.

(5) Amani, M.; Taheri, P.; Addou, R.; Ahn, G. H.; Kiriya, D.; Lien, D. H.; Ager, J. W., III; Wallace, R. M.; Javey, A. Recombination Kinetics and Effects of Superacid Treatment in Sulfur- and SeleniumBased Transition Metal Dichalcogenides. Nano Lett. 2016, 16 (4), 2786-91.

(6) Kim, H.; Lien, D. H.; Amani, M.; Ager, J. W., III; Javey, A. Highly Stable Near-Unity Photoluminescence Yield in Monolayer $\mathrm{MoS}_{2}$ by Fluoropolymer Encapsulation and Superacid Treatment. ACS Nano 2017, 11 (5), 5179-5185.

(7) Kiriya, D.; Hijikata, Y.; Pirillo, J.; Kitaura, R.; Murai, A.; Ashida, A.; Yoshimura, T.; Fujimura, N. Systematic Study of Photoluminescence Enhancement in Monolayer Molybdenum Disulfide by Acid Treatment. Langmuir 2018, 34 (35), 10243-10249.

(8) Schwermann, C.; Stiehm, T.; Tonndorf, P.; Schneider, R.; Schmidt, R.; Kern, J.; Michaelis de Vasconcellos, S.; Bratschitsch, R.; Doltsinis, N. L. Incorporation of Oxygen Atoms as a Mechanism for Photoluminescence Enhancement of Chemically Treated $\mathrm{MoS}_{2}$. Phys. Chem. Chem. Phys. 2018, 20 (25), 16918-16923.

(9) Roy, S.; Choi, W.; Jeon, S.; Kim, D. H.; Kim, H.; Yun, S. J.; Lee, Y.; Lee, J.; Kim, Y. M.; Kim, J. Atomic Observation of Filling Vacancies in Monolayer Transition Metal Sulfides by Chemically Sourced Sulfur Atoms. Nano Lett. 2018, 18 (7), 4523-4530.

(10) Kim, Y.; Lee, Y.; Kim, H.; Roy, S.; Kim, J. Near-Field Exciton Imaging of Chemically Treated $\mathrm{MoS}_{2}$ Monolayers. Nanoscale 2018, 10 (18), 8851-8858.

(11) Molas, M. R.; Gołasa, K.; Bala, Ł.; Nogajewski, K.; Bartos, M.; Potemski, M.; Babiński, A. Tuning Carrier Concentration in a Superacid Treated $\mathrm{MoS}_{2}$ Monolayer. Sci. Rep. 2019, 9, 1989.

(12) Kim, H.; Lee, T.; Ko, H.; Kim, S. M.; Rho, H. Influence of Chemical Treatment on Strain and Charge Doping in Vertically Stacked Monolayer-Bilayer $\mathrm{MoS}_{2}$. Appl. Phys. Lett. 2020, 117 (20), 202104.

(13) Li, Z.; Bretscher, H.; Zhang, Y.; Delport, G.; Xiao, J.; Lee, A.; Stranks, S. D.; Rao, A. Mechanistic Insight into the Chemical Treatments of Monolayer Transition Metal Disulfides for Photoluminescence Enhancement. Nat. Commun. 2021, 12, 6044.

(14) Bullock, J.; Kiriya, D.; Grant, N. E.; Azcatl, A.; Hettick, M.; Kho, T.; Phang, P.; Sio, H. C.; Yan, D.; Macdonald, D.; QuevedoLopez, M. A.; Wallace, R. M.; Cuevas, A.; Javey, A. Superacid Passivation of Crystalline Silicon Surfaces. ACS Appl. Mater. Interfaces 2016, 8 (36), 24205-11.

(15) Grant, N. E.; Niewelt, T.; Wilson, N. R.; Wheeler-Jones, E. C.; Bullock, J.; Al-Amin, M.; Schubert, M. C.; van Veen, A. C.; Javey, A.; Murphy, J. D. Superacid-Treated Silicon Surfaces: Extending the Limit of Carrier Lifetime for Photovoltaic Applications. IEEE J. Photovolt. 2017, 7 (6), 1574-1583.

(16) Pointon, A. I.; Grant, N. E.; Wheeler-Jones, E. C.; Altermatt, P. P.; Murphy, J. D. Superacid-Derived Surface Passivation for Measurement of Ultra-Long Lifetimes in Silicon Photovoltaic Materials. Sol. Energy Mater. Sol. Cells 2018, 183, 164-172.

(17) Pointon, A. I.; Grant, N. E.; Bonilla, R. S.; Wheeler-Jones, E. C.; Walker, M.; Wilshaw, P. R.; Dancer, C. E. J.; Murphy, J. D. Exceptional Surface Passivation Arising from Bis(trifluoromethanesulfonyl)-Based Solutions. ACS Appl. Electron. Mater. 2019, 1 (7), 1322-1329.

(18) Pointon, A. I.; Grant, N. E.; Pain, S. L.; White, J. T.; Murphy, J. D. Sub-2 cm/s Passivation of Silicon Surfaces by Aprotic Solutions. Appl. Phys. Lett. 2020, 116 (12), 121601.

(19) Grant, N. E.; Murphy, J. D. Temporary Surface Passivation for Characterisation of Bulk Defects in Silicon: A Review. Phys. Status Solidi RRL 2017, 11 (11), 1700243.

(20) Bonilla, R. S.; Hoex, B.; Hamer, P.; Wilshaw, P. R. Dielectric Surface Passivation for Silicon Solar Cells: A Review. Phys. Status Solidi A 2017, 214 (7), 1700293. 
(21) Grant, N. E.; Markevich, V. P.; Mullins, J.; Peaker, A. R.; Rougieux, F.; Macdonald, D.; Murphy, J. D. Permanent Annihilation of Thermally Activated Defects Which Limit the Lifetime of FloatZone Silicon. Phys. Status Solidi A 2016, 213 (11), 2844-2849.

(22) Piper, D. M.; Evans, T.; Leung, K.; Watkins, T.; Olson, J.; Kim, S. C.; Han, S. S.; Bhat, V.; Oh, K. H.; Buttry, D. A.; Lee, S.-H. Stable Silicon-Ionic Liquid Interface for Next-Generation Lithium-Ion Batteries. Nat. Commun. 2015, 6, 6230.

(23) Choi, J.; Zhang, H.; Du, H.; Choi, J. H. Understanding Solvent Effects on the Properties of Two-Dimensional Transition Metal Dichalcogenides. ACS Appl. Mater. Interfaces 2016, 8 (14), 8864-9.

(24) Lu, H.; Kummel, A.; Robertson, J. Passivating the Sulfur Vacancy in Monolayer $\mathrm{MoS}_{2}$. APL Mater. 2018, 6 (6), 066104.

(25) Fujisawa, K.; Carvalho, B. R.; Zhang, T.; Perea-López, N.; Lin, Z.; Carozo, V.; Ramos, S. L. L. M.; Kahn, E.; Bolotsky, A.; Liu, H.; Elías, A. L.; Terrones, M. Quantification and Healing of Defects in Atomically Thin Molybdenum Disulfide: Beyond the Controlled Creation of Atomic Defects. ACS Nano 2021, 15 (6), 9658-9669.

(26) Zhao, W.; Sun, J. Triflimide $\left(\mathrm{HNTf}_{2}\right)$ in Organic Synthesis. Chem. Rev. 2018, 118 (20), 10349-10392.

(27) Wang, R.; Xue, J.; Wang, K.-L.; Wang, Z.-K.; Luo, Y.; Fenning, D.; Xu, G.; Nuryyeva, S.; Huang, T.; Zhao, Y.; Yang, J. L.; Zhu, J.; Wang, M.; Tan, S.; Yavuz, I.; Houk, K. N.; Yang, Y. Constructive Molecular Configurations for Surface-Defect Passivation of Perovskite Photovoltaics. Science 2019, 366, 1509-1513.

(28) Zheng, W.; Zhu, Y.; Li, F.; Huang, F. Raman Spectroscopy Regulation in van der Waals Crystals. Photonics Res. 2018, 6 (11), 991.

(29) Zhao, W.; Ghorannevis, Z.; Amara, K. K.; Pang, J. R.; Toh, M.; Zhang, X.; Kloc, C.; Tan, P. H.; Eda, G. Lattice Dynamics in Monoand Few-Layer Sheets of $\mathrm{WS}_{2}$ and $\mathrm{WSe}_{2}$. Nanoscale 2013, 5, 96779683.

(30) Bolt, H. M.; Moretto, A.; Rietjens, I.; Papameletiou, D.; Klein, C. L. 1,2-Dichloroethane (Ethylene dichloride) Recommendation from the Scientific Committee on Occupational Exposure Limits; European Commission: Luxembourg, 2016; p 12.

(31) Prat, D.; Wells, A.; Hayler, J.; Sneddon, H.; McElroy, C. R.; Abou-Shehada, S.; Dunn, P. J. CHEM21 Selection Guide of Classicaland Less Classical-Solvents. Green Chem. 2016, 18, 288-296.

(32) Zheng, B.; Chen, Y. Controllable Growth of Monolayer $\mathrm{MoS}_{2}$ and $\mathrm{MoSe}_{2}$ Crystals Using Three-Temperature-Zone Furnace. IOP Conf. Ser.: Mater. Sci. Eng. 2017, 274, 012085.

(33) Shahzad, R.; Kim, T.; Kang, S.-W. Effects of Temperature and Pressure on Sulfurization of Molybdenum Nano-Sheets for $\mathrm{MoS}_{2}$ Synthesis. Thin Solid Films 2017, 641, 79-86.

(34) Niu, Y.; Gonzalez-Abad, S.; Frisenda, R.; Marauhn, P.; Drüppel, M.; Gant, P.; Schmidt, R.; Taghavi, N. S.; Barcons, D.; MolinaMendoza, A. J.; de Vasconcellos, S.; Bratschitsch, R.; Perez de Lara, D.; Rohlfing, M.; Castellanos-Gomez, A. Thickness-Dependent Differential Reflectance Spectra of Monolayer and Few-Layer $\mathrm{MoS}_{2}$, $\mathrm{MoSe}_{2}, \mathrm{WS}_{2}$ and $\mathrm{WSe}_{2}$. Nanomaterials 2018, 8 (9), 725.

(35) Yang, C.; Gao, Y.; Qin, C.; Liang, X.; Han, S.; Zhang, G.; Chen, R.; Hu, J.; Xiao, L.; Jia, S. All-Optical Reversible Manipulation of Exciton and Trion Emissions in Monolayer $\mathrm{WS}_{2}$. Nanomaterials 2020, 10 (1), 23

(36) Tanoh, A. O. A.; Alexander-Webber, J.; Xiao, J.; Delport, G.; Williams, C. A.; Bretscher, H.; Gauriot, N.; Allardice, J.; Pandya, R.; Fan, Y.; Li, Z.; Vignolini, S.; Stranks, S. D.; Hofmann, S.; Rao, A. Enhancing Photoluminescence and Mobilities in $\mathrm{WS}_{2}$ Monolayers with Oleic Acid Ligands. Nano Lett. 2019, 19 (9), 6299-6307.

(37) Bretscher, H.; Li, Z.; Xiao, J.; Qiu, D. Y.; Refaely-Abramson, S.; Alexander-Webber, J. A.; Tanoh, A.; Fan, Y.; Delport, G.; Williams, C. A.; Stranks, S. D.; Hofmann, S.; Neaton, J. B.; Louie, S. G.; Rao, A. Rational Passivation of Sulfur Vacancy Defects in Two-Dimensional Transition Metal Dichalcogenides. ACS Nano 2021, 15 (5), 87808789.

(38) Mak, K. F.; Lee, C.; Hone, J.; Shan, J.; Heinz, T. F. Atomically Thin $\mathrm{MoS}_{2}$ : A New Direct-Gap Semiconductor. Phys. Rev. Lett. 2010, 105 (13), 13605.
(39) McCreary, A.; Berkdemir, A.; Wang, J.; Nguyen, M. A.; Elías, A. L.; Perea-López, N.; Fujisawa, K.; Kabius, B.; Carozo, V.; Cullen, D. A.; Mallouk, T. E.; Zhu, J.; Terrones, M. Distinct Photoluminescence and Raman Spectroscopy Signatures for Identifying Highly Crystalline $\mathrm{WS}_{2}$ Monolayers Produced by Different Growth Methods. J. Mater. Res. 2016, 31 (7), 931-933.

(40) Lee, Y.; Jung, J. W.; Lee, J. S. Highly Electroconductive and Uniform $\mathrm{WS}_{2}$ Film Growth by Sulfurization of W Film Using Diethyl Sulfide. Mater. Chem. Front. 2021, 5 (9), 3692-3698.

(41) Di, X.; Wang, F.; Wei, J.; Shen, J.; Zhang, B.; Shan, X.; Lin, X.; Hao, Y.; Zhang, K. Controlled Synthesis of $\mathrm{WS}_{2}$ with Different Layers by Tuning Flow Rates. Mater. Sci. Eng., B 2020, 261, 114756.

(42) Zhao, Y.; Han, S.; Zhang, J.; Tong, L. Helicity-Resolved Resonant Raman Spectroscopy of Layered WS . J. Raman Spectrosc. 2021, 52 (2), 525-531.

(43) Foropoulos, J.; DesMarteau, D. D. Synthesis, Properties, and Reactions of $\mathrm{Bis}\left(\left(\right.\right.$ trifluoromethyl)sulfonyl) Imide, $\left(\mathrm{CF}_{3} \mathrm{SO}_{2}\right)_{2} \mathrm{NH}$. Inorg. Chem. 1984, 23 (23), 3720-3723.

(44) Reichardt, C.; Welton, T. Empirical Parameters of Solvent Polarity. Solvents and Solvent Effects in Organic Chemistry, 4th ed.; Wiley-VCH: Germany, 2011; p 455.

(45) Amani, M.; Taheri, P.; Addou, R.; Ahn, G. H.; Kiriya, D.; Lien, D. H.; Ager, J. W., III; Wallace, R. M.; Javey, A. Recombination Kinetics and Effects of Superacid Treatment in Sulfur- and SeleniumBased Transition Metal Dichalcogenides. Nano Lett. 2016, 16 (4), 2786-91.

(46) Yamada, Y.; Shinokita, K.; Okajima, Y.; Takeda, S. N.; Matsushita, Y.; Takei, K.; Yoshimura, T.; Ashida, A.; Fujimura, N.; Matsuda, K.; Kiriya, D. Photoactivation of Strong Photoluminescence in Superacid-Treated Monolayer Molybdenum Disulfide. ACS Appl. Mater. Interfaces 2020, 12 (32), 36496-36504.

(47) Cadiz, F.; Tricard, S.; Gay, M.; Lagarde, D.; Wang, G.; Robert, C.; Renucci, P.; Urbaszek, B.; Marie, X. Well Separated Trion and Neutral Excitons on Superacid Treated $\mathrm{MoS}_{2}$ Monolayers. Appl. Phys. Lett. 2016, 108, 251106.

(48) Yue, Y.-Y.; Wang, Z.; Wang, L.; Wang, H.-Y.; Chen, Y.; Wang, D.; Chen, Q.-D.; Gao, B.-R.; Wee, A. T S; Qiu, C.-W.; Sun, H.-B. Many-Particle Induced Band Renormalization Processes in Few- and Mono-Layer $\mathrm{MoS}_{2}$. Nanotechnology 2021, 32 (13), 135208.

(49) Dhakal, K. P.; Roy, S.; Yun, S. J.; Ghimire, G.; Seo, C.; Kim, J. Heterogeneous Modulation of Exciton Emission in Triangular $\mathrm{WS}_{2}$ Monolayers by Chemical Treatment. J. Mater. Chem. C 2017, 5 (27), 6820-6827.

(50) Grant, N. E.; Pointon, A. I.; Jefferies, R.; Hiller, D.; Han, Y.; Beanland, R.; Walker, M.; Murphy, J. D. Atomic Level Termination for Passivation and Functionalisation of Silicon Surfaces. Nanoscale 2020, 12, 17332-17341.

(51) Niewelt, T.; Steinhauser, B.; Richter, A.; Veith-Wolf, B.; Fell, A.; Hammann, B.; Grant, N. E.; Black, L.; Tan, J.; Youssef, A.; Murphy, J. D.; Schmidt, J.; Schubert, M. C.; Glunz, S. W. Reassessment of the Intrinsic Bulk Recombination in Crystalline Silicon. Sol. Energy Mater. Sol. Cells 2022, 235, 111467.

(52) Coffie, S.; Hogg, J. M.; Cailler, L.; Ferrer-Ugalde, A.; Murphy, R. W.; Holbrey, J. D.; Coleman, F.; Swadźba-Kwaśny, M. Lewis Superacidic Ionic Liquids with Tricoordinate Borenium Cations. Angew. Chem., Int. Ed. 2015, 54 (49), 14970-14973.

(53) Ji, W.; Zhao, Y.; Fahad, H. M.; Bullock, J.; Allen, T.; Lien, D. H.; De Wolf, S.; Javey, A. Dip Coating Passivation of Crystalline Silicon by Lewis Acids. ACS Nano 2019, 13 (3), 3723-3729.

(54) Harwell, J. R.; Baikie, T. K.; Baikie, I. D.; Payne, J. L.; Ni, C.; Irvine, J. T.; Turnbull, G. A.; Samuel, I. D. Probing the Energy Levels of Perovskite Solar Cells via Kelvin Probe and UV Ambient Pressure Photoemission Spectroscopy. Phys. Chem. Chem. Phys. 2016, 18 (29), 19738-45.

(55) Yang, C.; Du, P.; Dai, Z.; Li, H.; Yang, X.; Chen, Q. Effects of Illumination Direction on the Surface Potential of $\mathrm{CH}_{3} \mathrm{NH}_{3} \mathrm{PbI}_{3}$ Perovskite Films Probed by Kelvin Probe Force Microscopy. ACS Appl. Mater. Interfaces 2019, 11 (15), 14044-14050. 
(56) Tebyetekerwa, M.; Zhang, J.; Xu, Z.; Truong, T. N.; Yin, Z.; Lu, Y.; Ramakrishna, S.; Macdonald, D.; Nguyen, H. T. Mechanisms and Applications of Steady-State Photoluminescence Spectroscopy in Two-Dimensional Transition-Metal Dichalcogenides. ACS Nano 2020, 14 (11), 14579-14604.

(57) Chow, P. K.; Jacobs-Gedrim, R. B.; Gao, J.; Lu, T.-M.; Yu, B.; Terrones, H.; Koratkar, N. Defect-Induced Photoluminescence in Monolayer Semiconducting Transition Metal Dichalcogenides. ACS Nano 2015, 9 (2), 1520-1527.

(58) Plechinger, G.; Nagler, P.; Kraus, J.; Paradiso, N.; Strunk, C.; Schüller, C.; Korn, T. Identification of Excitons, Trions and Biexcitons in Single-Layer $\mathrm{WS}_{2}$. Phys. Status Solidi RRL 2015, 9 (8), 457-461.

(59) Kim, M. S.; Yun, S. J.; Lee, Y.; Seo, C.; Han, G. H.; Kim, K. K.; Lee, Y. H.; Kim, J. Biexciton Emission from Edges and Grain Boundaries of Triangular $\mathrm{WS}_{2}$ Monolayers. ACS Nano 2016, 10 (2), 2399-2405.

(60) Yamamoto, H.; Abbott, S.; Hansen, C. M. Consideration of Hansen Solubility Parameters - Part 3; Donor/Acceptor Interaction. Hansen Solubility Parameters 50th Anniversary Conference, York, UK, April 5-7, 2017; pp 22-36.

(61) Peimyoo, N.; Yang, W.; Shang, J.; Shen, X.; Wang, Y.; Yu, T. Chemically Driven Tunable Light Emission of Charged and Neutral Excitons in Monolayer $\mathrm{WS}_{2}$. ACS Nano 2014, 8 (11), 11320-11329. (62) Miao, X.; Tongay, S.; Petterson, M. K.; Berke, K.; Rinzler, A. G.; Appleton, B. R.; Hebard, A. F. High Efficiency Graphene Solar Cells by Chemical Doping. Nano Lett. 2012, 12 (6), 2745-50.

(63) Forsyth, M.; Hilder, M.; Zhang, Y.; Chen, F.; Carre, L.; Rakov, D. A.; Armand, M.; Macfarlane, D. R.; Pozo-Gonzalo, C.; Howlett, P. C. Tuning Sodium Interfacial Chemistry with Mixed-Anion Ionic Liquid Electrolytes. ACS Appl. Mater. Interfaces 2019, 11 (46), 43093-43106.

(64) Kessler, M.; Ohrdes, T.; Altermatt, P. P.; Brendel, R. The Effect of Sample Edge Recombination on the Averaged Injection-Dependent Carrier Lifetime in Silicon. J. Appl. Phys. 2012, 111 (5), 054508.

(65) Blum, A. L.; Swirhun, J. S.; Sinton, R. A.; Yan, F.; Herasimenka, S.; Roth, T.; Lauer, K.; Haunschild, J.; Lim, B.; Bothe, K.; Hameiri, Z.; Seipel, B.; Xiong, R.; Dhamrin, M.; Murphy, J. D. Interlaboratory Study of Eddy-Current Measurement of Excess-Carrier Recombination Lifetime. IEEE J. Photovolt. 2014, 4 (1), 525-531.

(66) Baikie, I. D.; Mackenzie, S.; Estrup, P. J. Z.; Meyer, J. A. Noise and the Kelvin Method. Rev. Sci. Instrum. 1991, 62 (5), 1326-1332.

(67) Gutmann, V. Solvent Effects on the Reactivities of Organometallic Compounds. Coord. Chem. Rev. 1976, 18 (2), 225-255.

(68) Beckett, M. A.; Strickland, G. C.; Holland, J. R.; Varma, K. S. A Convenient NMR Method for the Measurement of Lewis Acidity at Boron Centres: Correlation of Reaction Rates of Lewis Acid Initiated Epoxide Polymerizations with Lewis Acidity. Polymer 1996, 37 (20), 4629-4631.

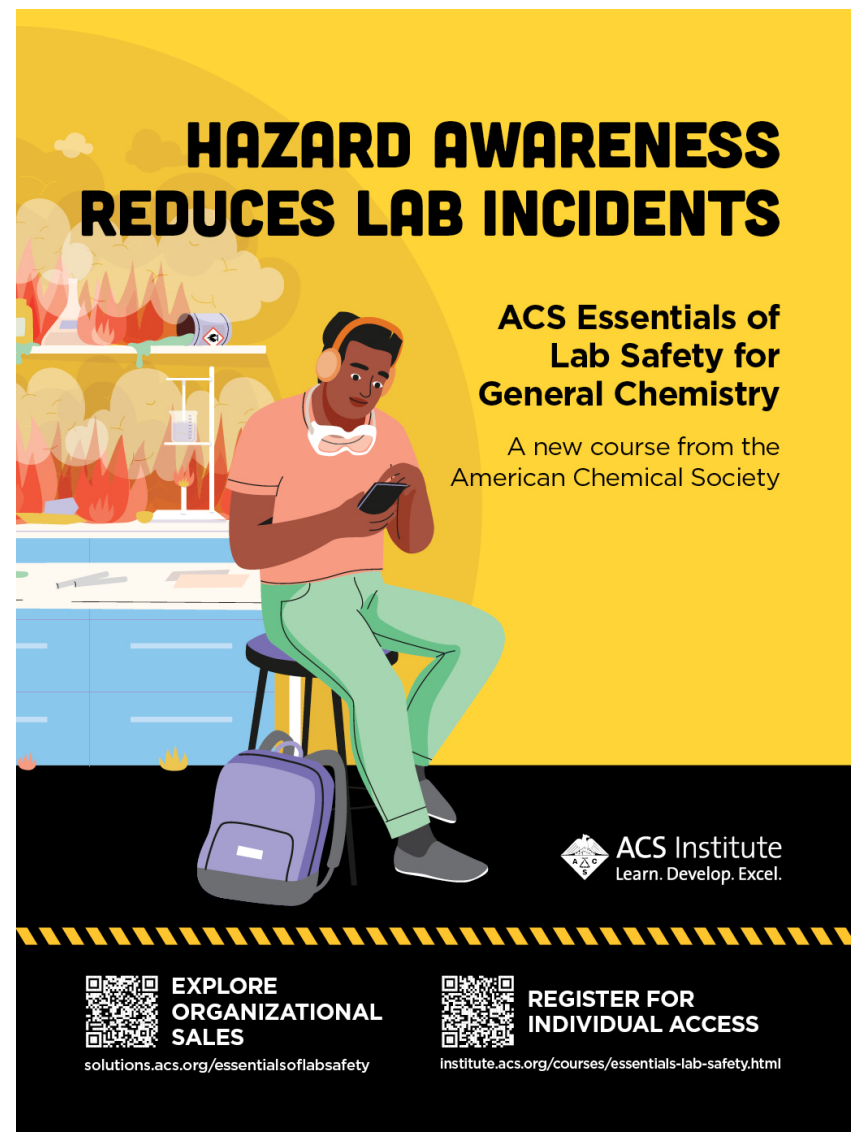

